\title{
THERMAL SHOCK STRUCTURAL ANALYSES OF A POSITRON TARGET
}

\author{
W. Stein, A. Sunwoo, LLNL, * Livermore, CA, USA \\ V.K. Bharadwaj, D.C. Schultz, J.C. Sheppard, SLAC, ${ }^{*}$ Stanford, CA, USA
}

\section{Abstract}

In the positron source of the Stanford Linear Collider (SLC), the electron beam collides with a tungsten-rhenium target. As the beam passes into the material, thermal energy is created that heats the material to several hundred degrees centigrade on a time scale of nanoseconds. The heating of the material results in thermal stresses that may be large enough to cause material failure. The analyses calculate the thermal shock pressure and stress pulses as they move throughout the material due to the rapid energy deposition. Failure of the target occurred after three years of operation with an elevated power deposition toward the end of the three years. The calculations were made with the LLNL coupled heat transfer and dynamic solid mechanics analysis codes, TOPAZ3D and DYNA3D, and the thermal energy deposition was calculated with the SLAC Electron Gamma Shower (EGS) code simulating the electron-induced cascade. Material fatigue strength, experimentally measured properties for the non-irradiated and irradiated material, as well as the calculated stress state are evaluated in assessing the cause for the target failure.

\section{INTRODUCTION}

The SLC positron target was operated for several years with gradually increasing electron beam power. Inspection of the target showed considerable damage on the backside of the target in the region where the beam exits the target $[1,2]$. The damage observed consisted of approximately one millimeter of missing material and cracks propagating in various directions into the target.

In an attempt to determine the cause of the material loss and cracks, a dynamic stress analysis was undertaken to model the pressure-induced stress waves in the material.

\section{BACKGROUND}

The SLC target consists of a hockey puck-shaped piece of Tungsten and 25\% Rhenium (W25Re). Figure 1 shows the geometry of the target. The target consists of a 2.5inch diameter disc of W25Re with a six-radiation length thickness of 0.81 inches. The target is cooled by two cooling tubes, and they maintain the tungsten-rhenium target at a nominal $115 \mathrm{C}$. The individual spots on the target heat up an additional maximum amount of approximately $200 \mathrm{C}$ and relax down to the $115 \mathrm{C}$

\footnotetext{
* Work supported by the U.S. Department of Energy under Contract Nos. DE-AC03-76SF00515 (SLAC) and by Lawrence Livermore National Laboratory under Contract W-7405-Eng-48. Neither the U.S. Government nor the University of California makes any warranty or assumes any legal liability for the accuracy, completeness or usefulness of the work performed.
}

temperature before another electron bunch impinges [3]. During operation the target is rotated in a way that the impinging beam pulses strike a different part of the target after every pulse.

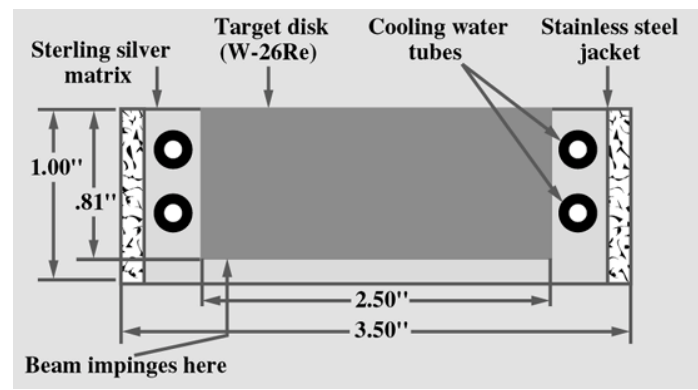

Figure 1: SLC target geometry.

\subsection{Electron beam energy deposition}

The electron beam of $4.0 \mathrm{e}+10$ electrons/bunch at $33 \mathrm{Gev}$ impinging on the target deposits $5 \mathrm{~kW}$ of power into the target at a frequency of 120 hertz. The beam has a Gaussian radial distribution with a spot size of $0.8 \mathrm{~mm}$. The SLAC EGS code was used to calculate the energy deposition profile in the target. The energy deposition [4] is very low as the beam enters the target and increases to a maximum as the beam exits the back of the target (see Fig. 2).

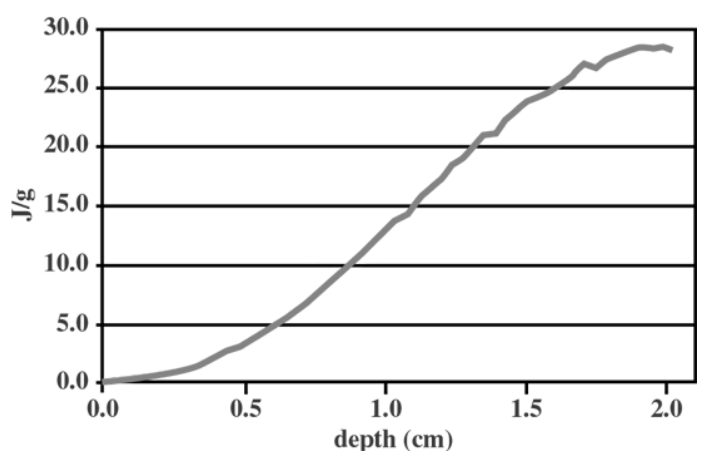

Figure 2: Beam centerline energy deposition, $\mathrm{J} / \mathrm{g}$, versus depth into target, $\mathrm{cm}$.

\section{ANALYSES}

A dynamic three-dimensional finite element structural analysis was made for the target. The analysis involved modeling the beam energy deposition and determining the temperature rise from thermal heat capacity data.

The thermal results are coupled into a dynamic structural analysis by using a thermal coefficient of expansion, which determines the material volume expansion. 
From the volume expansion, the material stress conditions are calculated from the material stress versus strain properties.

\subsection{Thermal energy calculations}

The beam energy is deposited in the target in picoseconds and results in a temperature profile in the material over that time scale. The LLNL three dimensional finite element heat transfer code, TOPAZ3D [5], was used to calculate the temperature history in the target from input of energy over the beam impacted region of the target. From heat capacity data for the material, the temperature is then calculated. The calculated temperature profile in the target is shown in Figure 3.

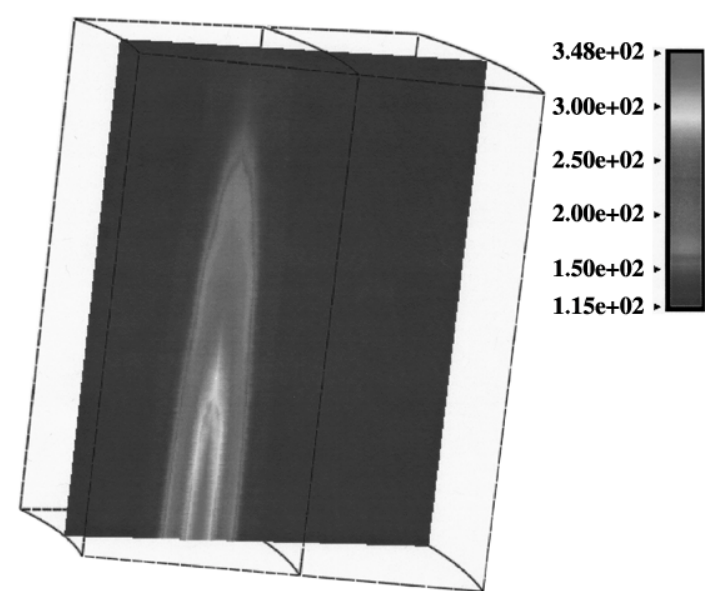

Figure 3: Temperature, C, profile in target.

The timescales of this problem are such that the beam heats up the target material essentially instantaneously. Thermal stress wave effects are over after about 1.0e- 05 seconds and the material assumes a static stress state. The temperature profile of a spot requires about 1 second to relax down to initial temperature.

\subsection{Structural analyses calculations}

The dynamic structural modeling used the LLNL DYNA3D code [6] which requires a calculational mesh of sufficient detail to model the physics of the problem. The mesh shown in Figure 4, models a section of the target disc, including the silver surrounding the tungstenrhenium disc.

The materials in the problem are modeled with a temperature dependent elastic-plastic material model. Properties specified include the elastic modulus, Poisson's ratio, secant coefficient of thermal expansion, yield stress, and plastic modulus. For these analyses, the properties used are shown in Table 1.
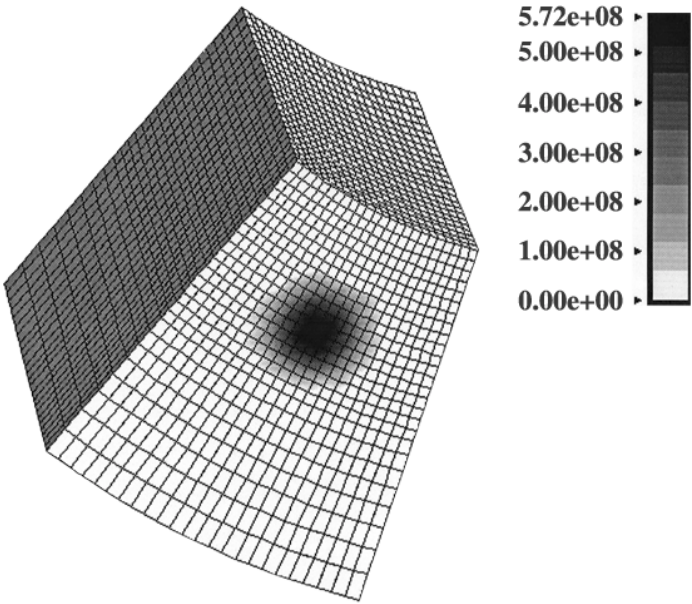

Figure 4: Maximum Von Mises stress, Pa, profile.

Table 1: WRe structural material properties.

\begin{tabular}{|l|l|l|l|}
\hline Property & $\mathbf{0 . 0}$ C & $\mathbf{5 0 0}$ C & $\mathbf{1 0 0 0}$ C \\
\hline Modulus, $\mathrm{Pa}$ & $4.3 \mathrm{e}+11$ & $4.0 \mathrm{e}+11$ & $3.95 \mathrm{e}+11$ \\
\hline Poisson ratio & 0.28 & 0.28 & 0.28 \\
\hline $\begin{array}{l}\text { Thermal Exp. } \\
\text { Coef. } 1 / \mathrm{C}\end{array}$ & $6.7 \mathrm{e}-6$ & $7.1 \mathrm{e}-6$ & $7.95 \mathrm{e}-6$ \\
\hline Yield, $\mathrm{Pa}$ & $1.6 \mathrm{e}+9$ & $1.3 \mathrm{e}+9$ & $9.0 \mathrm{e}+8$ \\
\hline
\end{tabular}

The structural response of the target is so fast, less than $1.0 \mathrm{e}-5$ seconds, that only the stress waves for one spot location needs to be analyzed. Before an adjacent spot is impinged upon, 1/120 seconds have elapsed and the shock pulses have dissipated.

The rapid beam energy deposition results in stresses that are greatest at the target location where the beam shower exits the target. Von Mises stress is a good measure of the proximity to failure of a material with values below material yield stress indicating elastic behavior. Figure 4 shows the calculated maximum stress. A plot of Von Mises stress over time (see Fig. 5) shows how the stress varies on the backside of the target.

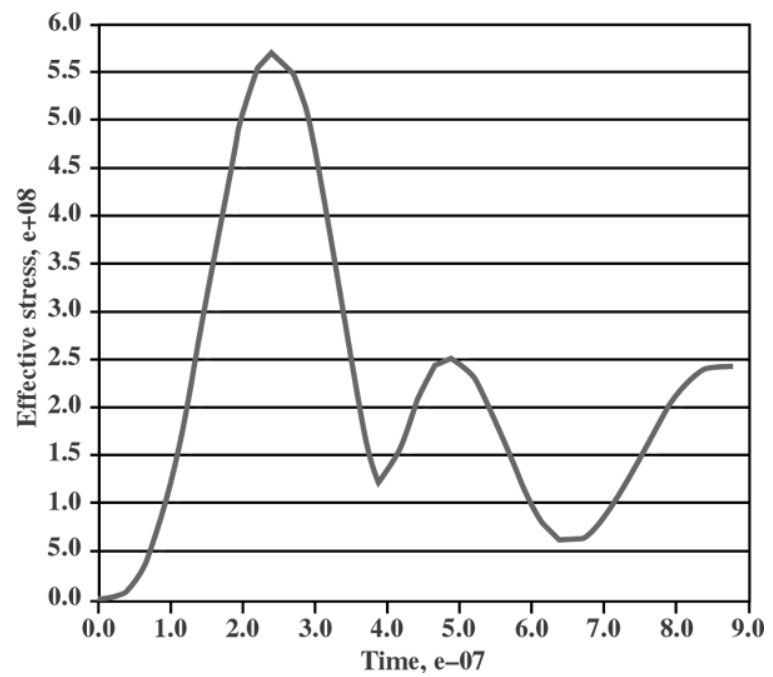

Figure 5: Peak Von Mises stress, Pa, versus time, s, plot. 


\subsection{Quasi-static stress calculations}

After the stress waves dissipate, a steady state temperature field exists in the material before diffusion appreciably affects the temperature. For this state, Figure 6 shows the peak Von Mises stresses in the target. The stresses are about one half those under dynamic conditions.

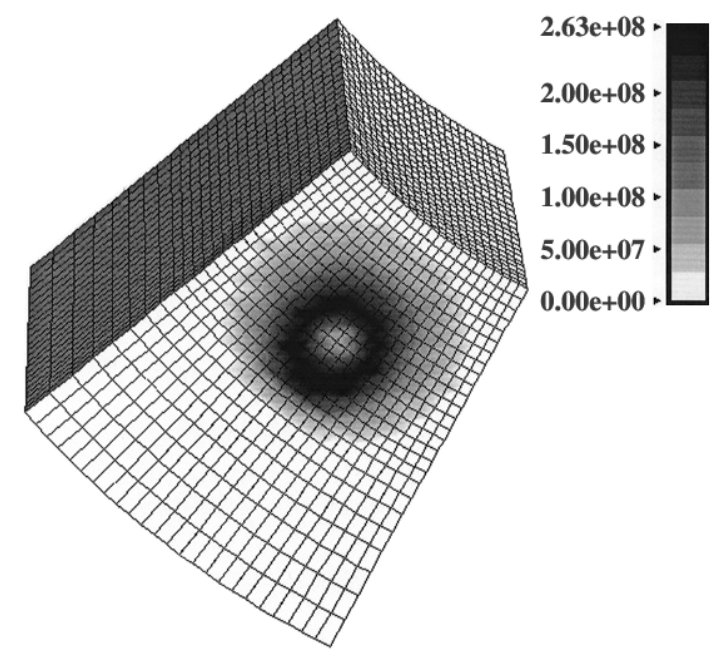

Figure 6: Peak quasi-static Von Mises stress, Pa.

\section{CONCLUSIONS}

The analyses show that stresses do not exceed material yield stress. After many beam pulses, fatigue failure may occur. A general criteria of failure due to a Von Mises stress of $50 \%$ of tensile strength may apply to this target material. For a tensile strength of $1.1 \mathrm{e}+9 \mathrm{~Pa}(160 \mathrm{ksi})$ (at $348 \mathrm{C}$ ), the calculated peak Von Mises stress of $5.7 \mathrm{e}+8 \mathrm{~Pa}$ ( $83 \mathrm{ksi})$ is at $52 \%$ of the tensile stress.

Material cracks, once formed, may also contribute to part failure by raising temperatures and reducing yield stress. Radiation damage also may make the material more brittle and susceptible to failure.

\section{REFERENCES}

[1] S. Maloy, et al., "SLC Target Analysis," LANL LAUR-01-1913, June 2001.

[2] V.K. Bharadwaj, et al., "Analysis of Beam-Induced Damage to the SLC Positron Production Target," Proc. PAC 2001, Chicago, IL, June 2001. (WPAH019)

[3] W. Stein, "Thermal Shock Structural Analyses of the SLC Positron Target," LLNL, Livermore, CA, UCRL-PRES-143893, April 24, 2001.

[4] Private communication with D.C. Schultz, SLAC, Stanford, CA, May 2001.

[5] A.B. Shapiro, "TOPAZ3D-A Three-dimensional Finite Element Heat Transfer Code," LLNL, Livermore, CA, UCID-20484, August 1985.

[6] J.O. Hallquist, R.G. Whirley, "DYNA3D User's Manual," LLNL, Livermore, CA, UCID-19592, May 1989. 